\title{
Becker muscular dystrophy in Indian patients: Analysis of dystrophin gene deletion patterns
}

\author{
Rashna S. Dastur, Pradnya S. Gaitonde, Satish V. Khadilkar ${ }^{1}$, Jayshree J. Nadkarni \\ Department of Neuropathology and Applied Biology, ${ }^{1}$ Department of Neurology, Bombay Hospital, Mumbai, India
}

\begin{abstract}
Background: Becker muscular dystrophy (BMD) is caused by mutations in the dystrophin gene with variable phenotypes. Becker muscular dystrophy patients have low levels of nearly full-length dystrophin and carry in-frame mutations, which allow partial functioning of the protein. Aim: To study the deletion patterns of BMD and to correlate the same with reading frame rule and different phenotypes. Setting: $A$ tertiary care teaching hospital. Design: This is a prospective hospital-based study. Materials and Methods: Thirty-two exons spanning different "hot spot" regions using Multiplex PCR techniques were studied in 347 patients. Two hundred and twenty-two showed deletions in one or more of the 32 exons. Out of these, 46 diagnosed as BMD patients were analyzed. Results: Forty-six BMD patients showed deletions in both regions of the dystrophin gene. Out of these $89.1 \%$ $(41 / 46)$ were in-frame deletions. Deletions starting with Exon 45 were found in $76.1 \%(35 / 46)$ of the cases. Mutations in the majority of cases i.e. 39/46 (84.8\%) were seen in 3' downstream region (Exon 45-55, distal rod domain). Few, i.e. $5 / 46(10.8 \%)$ showed deletions in 5 ' upstream region (Exons 3-20, N-terminus and proximal rod domain) of the gene, while in $2 / 46(4.4 \%$ ) large mutations (>40 bp) spanning both regions (Exons 3-55) were detected. Conclusion: This significant gene deletion analysis has been carried out for BMD patients particularly from Western India using 32 exons.
\end{abstract}

Key words: Becker muscular dystrophy, dystrophin gene, in-frame deletions, out-frame deletions

\section{Introduction}

Severe Duchenne and milder Becker muscular dystrophy (DMD/BMD) are neuromuscular disorders caused by mutations (mainly intragenic exon deletions) in the dystrophin gene. ${ }^{[1]}$ Dystrophin is a large gene $(2.4 \mathrm{Mb}$ ) with 79 exons (covering only $0.6 \%$ ) with a coding sequence of $14 \mathrm{~kb} \cdot{ }^{[2]}$ Clinically, BMD (incidence approximately $1 / 12,000$ ), is similar to DMD but milder with late onset and loss of ambulation. ${ }^{[3]}$

Becker muscular dystrophy patients carry mainly in-frame deletions, which allow partial functioning of dystrophin protein and hence results in a milder phenotype, whereas in DMD, frame-shift results in loss of dystrophin. ${ }^{[4]}$ Phenotypic differences between BMD and DMD can be explained by the reading frame rule in $90 \%$ of reported cases. ${ }^{\left[{ }^{[3}\right.}$ In this study we report 46 cases of BMD patients from Western India with 89.1\% following the reading frame rule.

\section{Materials and Methods}

A total of 347 samples with clinical diagnosis of $\mathrm{DMD} / \mathrm{BMD}$ were received over the past three years in our department, for deletion studies of 32 exons of dystrophin gene. Out of which, 222 showed deletions of one or more exons. Among the patients showing deletions, 176 were DMD while 46 showed typical BMD phenotype i.e. patients were ambulant above the age of 12-13 years. Except for 19 familial cases, all DMD cases were sporadic and two out of 46 cases of BMD were familial.

Genomic DNA was extracted using Sigma NA 2020 kit from peripheral blood (collected in EDTA bulbs). Multiplex PCR was carried out using multiplex PCR kit (Qiagen). A total of 32 exons (Pm, 1-13,19-21,43-55,60) were screened using three primer sets, 13-plex PCR, 9-plex PCR and 10-plex PCR..$^{[6-8]}$

Conditions for all three sets of primers were: denaturation at $94^{\circ} \mathrm{C}$ for $30 \mathrm{~s}$, annealing at $52^{\circ} \mathrm{C}$ for $30 \mathrm{~s}$, extension at $65^{\circ} \mathrm{C}$ for $4 \mathrm{~min}$, with a total of 26 cycles. ${ }^{[9]}$ The PCR products $(25 \mu \mathrm{l})$ were separated using $3 \%$ NuSieve agarose gel (Cambrex, USA) stained with ethidium bromide and bands observed on gel documentation system (Vilber Lourmat, France). 
The DMD/BMD exon deletions obtained by multiplex PCR methods for 32 exons were subjected to the checker of reading frame from Leiden Database Muscular Dystrophy. ${ }^{[10]}$ The data collected on the basis of "checker list" enabled us to predict the shift in translational reading frame.

\section{Results}

A total of 222 patients showed deletions of one or more exons, out of these 46 were BMD. In-frame deletions were seen in $89.1 \%$ (41/46) of the BMD cases, while $6.5 \%$ (3/46) were out-frame though clinically the symptoms corresponded to BMD phenotype and 4.4\% (2/46) could not be classified as either [Table 1].

These were classified on the basis of their neurological deficit i.e. extent of muscle weakness, age of onset and ability to walk after the age of 12-13 years. These 46 patients displayed deletions in different exons in both regions of the dystrophin gene [Table 2]. The majority $(84.8 \%)$ had deletions in the central rod domain between Exons 45-55 and out of these 76.1\% deletions started with Exon 45.

In the above series of BMD patients, five patients showed in-frame deletions in the upstream region (5' end) of the gene (between Exons 3 and 20), four were between ages 15 and 17 years and one aged 22 years. Clinical symptoms of the four patients were nearly similar with creatinine phosphokinase (CPK) ranging from 3000 to 8000 IU, hypertrophy of calves, Gower's sign, difficulty in climbing, but ambulant. The oldest patient aged 22, showed deletions of Exons (14-) 17-20, was non-ambulatory by age 19 years.

\begin{tabular}{lccc}
\hline \multicolumn{4}{c}{ Table 1: Total number of DMD/BMD patients with in-/out-frame } \\
& deletions & \\
\hline Deletions & DMD & BMD & Total \\
In-frame & $16(9.1)$ & $41(89.1)$ & \\
Out-frame & $155(88.1)$ & $3(6.5)$ & \\
In-/out-frame & $5(2.8)$ & $2(4.4)$ & \\
& 176 & 46 & 222 \\
\hline
\end{tabular}

Figure in parenthesis are in \%
Two patients aged 15 and 18 showed deletions spanning both the regions involving absence of $>40$ exons [Table 2]. The 15-year-old had an out-frame deletion (Exon 3-43) with onset at age 12 years, CPK-21,800 IU, calf hypertrophy, Gower's sign, limb girdle weakness and EMG reporting myopathic changes. Though the symptoms were severe the patient could still walk without support. He had deletions of both the actin- binding and the rod domain (Exons 3-43) showing out-frame deletions but with a BMD phenotype. This was considered as more severe BMD type. The other 18-year-old patient showed in/out-frame deletions of Exons 13-55(-59) with less severe symptoms (CPK-9,000 IU) and late onset at age 14 years after which he presented with enlarged calves, brachioradialar weakness of hips and shoulder girdle and difficulty in climbing and running, but could walk without support.

A total of 39 patients (84.8\%) showed deletions in the downstream region of the gene. Out of these, 36 patients were showing in-frame mutations.

A maximum number of patients (35 i.e. 76.1\%) in BMD showed deletions starting with Exon 45. Out of these only one patient with deletions of Exons 45-55(-59), could be either out or in-frame while the rest were all in-frame. This patient was 19 years old with an onset at age 16 years, his CPK was 1132 IU and he showed hypertrophy of calves, Gower's signs and difficulty in climbing stairs.

Deletions starting with Exon 45 were seen in the remaining 34 patients with in-frame pattern. In 14 patients of the same group, the ages varied from 12 to 44 years with $78.5 \%$ being above the age of 18 years. The age of onset for three of the patients was in their twenties. All these patients showed hypertrophy of calves, had difficulty in climbing stairs, Gower's signs and four had weakness and wasting of limbs. The CPK ranged from 1000 to 11,885 IU. All were ambulant and only one patient (44 years old) had a family history of maternal uncle being affected.

The other 20 patients with in-frame deletions of Exons 45-48, 45-49 (BMD Patient 2 in Figure 1A,C) and 45-53

\begin{tabular}{|c|c|c|c|c|c|}
\hline Exons deleted & In-/out-frame & No. of pts. (46) & $\begin{array}{l}\text { Age range } \\
\text { (in years) }\end{array}$ & $\begin{array}{c}\text { Age of onset } \\
\text { (range in years) }\end{array}$ & Percentage \\
\hline $3-4$ & In-frame & 2 & $16-17$ & $13-15$ & $4.3(2 / 46)$ \\
\hline $3-13(-16 ?)$ & In-frame & 2 & 15 & $9-11$ & $4.3(2 / 46)$ \\
\hline (?14-) 17-20 & In-frame & 1 & 22 & 9 & $2.2(1 / 46)$ \\
\hline $3-43$ & Out-frame & 1 & 15 & 12 & $2.2(1 / 46)$ \\
\hline $13-55(-59 ?)$ & In-/out-frame & 1 & 18 & 14 & $2.2(1 / 46)$ \\
\hline $45-47$ & In-frame & 14 & $12-44$ & $5-26$ & $30.4(14 / 46)$ \\
\hline $45-48$ & In-frame & 7 & $16-27$ & $9-22$ & $16.2(7 / 46)$ \\
\hline $45-49$ & In-frame & 9 & $12-23$ & $8-14$ & $19.6(9 / 46)$ \\
\hline $45-53$ & In-frame & 4 & $13-33$ & $9-10$ & $8.7(4 / 46)$ \\
\hline $45-55(-59 ?)$ & Out-/in-frame & 1 & 19 & 16 & $2.2(1 / 46)$ \\
\hline $46-47$ & Out-frame & 2 & $18-23$ & $11-17$ & $4.3(2 / 46)$ \\
\hline 48 & In-frame & 2 & $18-29$ & $12-14$ & $4.3(2 / 46)$ \\
\hline
\end{tabular}


(BMD Patient 1 in [Figure 1A-C]) have ages ranging from 12 to 33 years with $60 \%$ above the age of 18 years. The age of onset also varies from age 8 to 22 years. Calf hypertrophy manifested in 19 of them, 10 had difficulty in climbing stairs, $40 \%$ showed Gower's signs and three

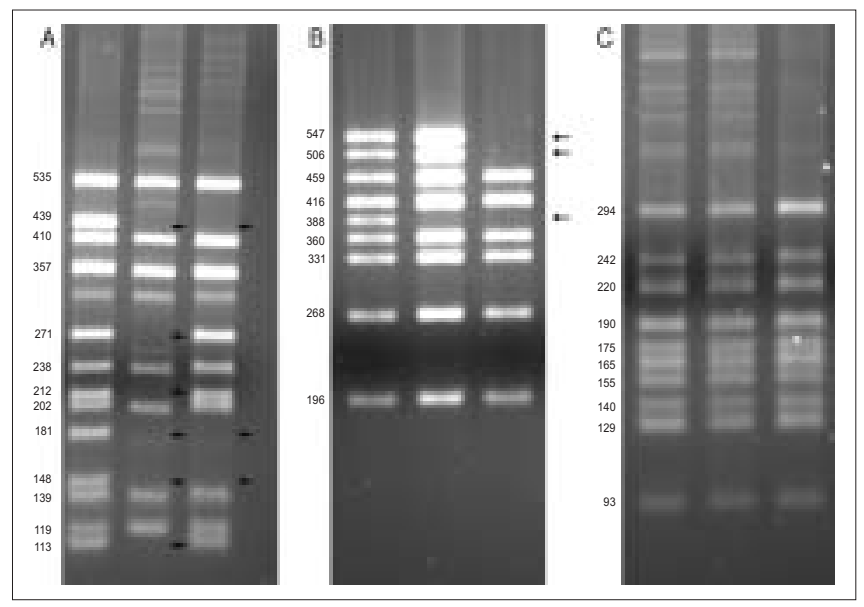

Figure 1: (A) Gel electrophoresis: Set of 13 exons[6] of Dystrophin gene, from left lane: control sample; BMD patient-1 showing deletions of exons 52(113bp), 46(148bp), 47(181bp), 53(212bp), 50(271bp), 49(439bp) (arrows); BMD patient-2 showing deletions (arrows) of exons $46(148 \mathrm{bp})$, 47(181bp) and 49(439bp). (B) Set of 9 exons[7] of Dystrophin gene; from left: control sample; BMD patient-1 showing deletions (arrows) of exons $52(388 \mathrm{bp}), 48(506 \mathrm{bp})$ and $45(547 \mathrm{bp})$. (C) Set of 10 exons of Dystrophin gene; from left lane: control sample; BMD patient-1 and 2 showing no deletion in the 10 exon set

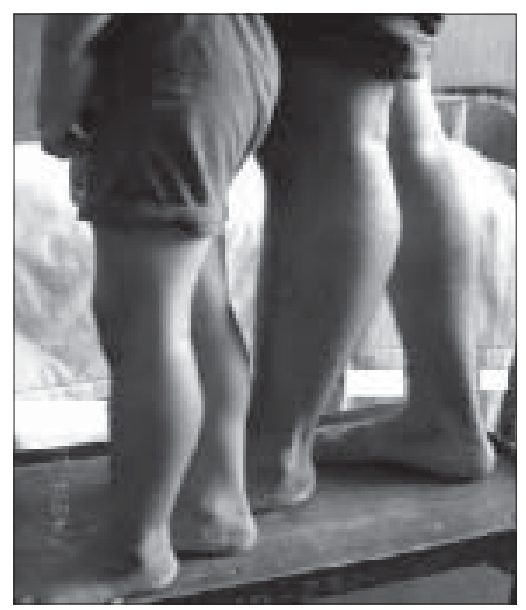

Figure 2: Phenotypic variability in a family: index case had DMD phenotype while maternal uncle had BMD phenotype showed mild limb girdle weakness. All of them were ambulant with two having a family history with affected maternal uncle and the other with affected brother and nephew. In the case of the patient with a family history of affected brother and nephew, the nephew (age six years) was an index case with DMD phenotype. Both showed large calves [Figure 2] and in-frame deletions of Exons 45-48 but manifested with variable phenotype.

Single Exon 48 deletion resulting in an in-frame mutation were seen in two patients aged 18 and 29 years. The CPK of these patients was 4004 and 3800 IU respectively and both were with hypertrophy of calves and difficulty in climbing stairs.

Out-frame deletion of Exons 46-47 which were most common in our DMD patients [Table 3], were also seen in two of our BMD patients aged 18 and 23 years. Both could walk without support, the onset being at age 15 and 17 years respectively. The 23-year-old mainly complained of weakness, while the 18-year-old exhibited both hypertrophy of calves and Gower's signs thereby suggesting BMD phenotypes with discordant out-frame deletions.

\section{Discussion}

In our study of 222 patients showing deletions, 88.1\% (out-frame) of DMD and 89.1\% (in-frame) of BMD, conformed to the reading frame rule [Table 1].

This was in accordance with world literature except for a North Indian study, which showed a higher percentage i.e. more than $30 \%$ deviation from the reading frame rule..$^{[11,12]}$

The majority of the discrepancies had only been confirmed at DNA level, as was the case in our study. It was likely that at the RNA level, due to variable levels of alternative splicing one or more exons may either restore or disrupt the reading frame. ${ }^{[11]}$ It was not yet clear why the exon-skipping mechanism appeared more efficient for some patients than others. This may be due to the exact location of the break points within the intron where certain intronic elements required were lost or maintained for the proper inclusion of certain exons. ${ }^{[11]}$ This may also help to explain the overlap of deleted exon range seen in some of our patients [Table 3].

\begin{tabular}{|c|c|c|c|c|c|}
\hline & & 3: Deletion & both in BMD and DMD & & \\
\hline \multirow[t]{2}{*}{ Exons deleted } & \multirow[t]{2}{*}{ In-/out-frame } & \multicolumn{2}{|c|}{ BMD } & \multicolumn{2}{|c|}{ DMD } \\
\hline & & No. of pts & Age range (in years) & No. of pts. & Age range (in years) \\
\hline $3-13(-16 ?)$ & In-/out-frame & 2 & 15 & 2 & $6-10$ \\
\hline $17-20$ & In-frame & 1 & 22 & 1 & 8 \\
\hline $45-47$ & In-frame & 14 & $12-44$ & 2 & $4-8$ \\
\hline $45-48$ & In-frame & 7 & $16-27$ & 1 & 6 \\
\hline $45-49$ & In-frame & 9 & $12-23$ & 1 & 10 \\
\hline $45-55(-59 ?)$ & Out-/in-frame & 1 & 19 & 1 & 5 \\
\hline $46-47$ & Out-frame & 2 & $18-23$ & 18 & $2.5-11$ \\
\hline 48 & In-frame & 2 & $18-29$ & 2 & $5-14$ \\
\hline
\end{tabular}


Different phenotypes were sometimes found within families as seen in an index case of a six-year-old boy with DMD phenotype and his maternal uncle with BMD phenotype [Figure 2] both with in-frame deletions of Exons 45-48. Different phenotypes have been reported in families even where deletion break points were identical. ${ }^{[1]}$ The mechanism of pre-mRNA splicing seems to be complex and regulated by many factors, especially in the case of a large complex gene like dystrophin. Each individual may express these factors at different levels, which may explain the observed differences to some extent. ${ }^{[13]}$

As reported earlier in in-frame mutations the phenotype was to some extent determined by the location and size of deletion. ${ }^{[14]}$ Large deletions spanning $>35$ exons usually were associated with DMD phenotype. ${ }^{[14-17]}$ Our two BMD patients with deletions extending $>40$ exons were exceptions. One of them aged 18 years showed deletions spanning most of the rod region (Exons 13-55(-59?)), though phenotypically he presented with milder symptoms. The other patient had deletions of both actin-binding and rod domain (Exons 3-43) showing out-frame deletions but with a BMD phenotype. Out of all the patients studied, both DMD and BMD, we had four patients with these large deletions i.e. absence of $>40$ exons, including both regions. Two were BMD (described above) and two DMD with out-frame deletions spanning Exons 2-44 and 3-45 aged two years and four years respectively with severe DMD phenotype. From India, there are no such reported cases of BMD with large-scale $(>40)$ exon deletions.

Many cases of BMD are not reported from India, especially Western region. The data is mainly from north and eastern India, where gene deletion analysis of mainly 18 exons in both DMD and BMD were reported. ${ }^{[12,18-23]}$ The majority of the BMD cases reported from India and abroad showed deletions in the major "hot spot" region between Exons 45-53, which is in accordance with our data [Table 2]..$^{[6,12,18-23]}$

In our analysis, BMD patients were exclusively analyzed on the deletion patterns obtained using 32 exons multiplex reaction. A majority of the upstream exons between 1-21 were included in this study. The in-frame and out-frame patterns were determined. ${ }^{[10]}$ The exact extent of deletion at the 3' end of patients having deletions from 3-13, 13-55, 45-55 and at the 5' end exons of patient with exon deletions 17-20 could not be accurately determined. This was due to the fact that the exons preceding or following the deletions were not included in the 32 exons analyzed. The in-frame classification will not change for 3-13 and 17-20 as all the exons proceeding and preceding the range also result in in-frame mutations. Two BMD patients who showed exon deletions between 13-55 and 45-55 could not be confirmed as in- or out-frame mutations, because after
Exon 55, Exon 56 to Exon 59 (not constituents of the 32 exons analyzed) give alternate out-/in-frame deletion patterns. ${ }^{[10]}$

Thus, in conclusion, this study revealed a significant correlation between clinical severity, molecular deletions and translational reading frame hypothesis with a few exceptions not conforming to the same. These exceptions are only at a DNA level and in fact concur with the reading frame on RNA studies. Therefore, it is advisable to confirm the mutations at the RNA level.

\section{Acknowledgments}

Our thanks to Dr. Madhuri Hegde, Assistant Professor, Medical Genetics Laboratory Baylor College of Medicine, Houston, for her help in modifications of the multiplex PCR protocol.

\section{References}

1. Cohn RD, Campbell KP. Molecular basis of muscular dystrophies. Muscle Nerve 2000;23:1456-71.

2. Koenig M, Monaco AP, Kunkel LM. The complete sequence of dystrophin predicts a rod-shaped cytoskeletal protein. Cell 1988;53:219-28.

3. Emery AE. The muscular dystrophies. Lancet 2002;359:687-95.

4. Koenig M, Beggs AH, Moyer M, Scherpf S, Heindrich K, Bettecken T, \& al. The molecular basis for Duchenne versus Becker muscular dystrophy: Correlation of severity with type of deletion. Am J Hum Genet 1989;45:498-506.

5. Monaco AP, Bertelson CJ, Liechti-Gallati S, Moser H, Kunkel LM. An explanation for the phenotype differences between patients bearing partial deletions of the DMD locus. Genomics 1988;2:90-5.

6. Beggs AH, Koenig M, Boyce FM, Kunkel LM. Detection of $98 \%$ of DMD/BMD gene deletions. Hum Genet 1990;86:45-8.

7. Chamberlain JS, Gibbs RA, Ranier JE, Nguyen PN, Caskey CT. Deletion screening of the Duchenne muscular dystrophy locus via multiplex DNA amplification. Nucl Acid Res 1988;16:11141-56.

8. Den Dunnen JT. Leiden Muscular Dystrophy pages. Center for human and Clinical Geneties, Leiden University Medical Center. DNA based diagnostic techniques for DMD/BMD Deletion Detection using multiplex PCR [updated on 2005 Mar 16]. Available from: http://www.dmd.nl/.

9. Chamberlain JS, Gibbs RA, Ranier JE, Caskey CT. Multiplex PCR for the diagnosis of Duchenne muscular dystrophy. In: PCR protocols: A guide to methods and applications. Innis MA, Gelfand DH, Sninsky J, White TJ, editors. New York, London; Academic Press; 1990; 272-81.

10. Den Dunnen JT. Leiden Muscular Dystrophy pages. Center for Human and Clinical Geneties, Leiden University Medical Center. DMD exonic deletions/duplications reading-frame checker 1.6 [updated on 2005 Mar 16]. Available from: http://www.dmd.nl/.

11. Aartsma-Rus A, Van Deutekom JC, Fokkema IF, Van Ommen G.J, Den Dunnen JT. Entries in the Leiden Duchenne Muscular Dystrophy mutation database: An overview of mutation types and paradoxical cases that confirm the reading frame rule. Muscle Nerve 2006;34:135-44.

12. Pandey GS, Kesari A, Mukherjee M, Mittal RD, Mittal B. Re-evaluation of reading frame shift hypothesis in Duchenne and Becker muscular dystrophy. Neurology India 2003;51:367-9.

13. Ramelli GP, Joncourt F, Luetschg J, Weis J, Tolnay M, Burgunder JM. Becker muscular dystrophy with marked divergence between clinical and molecular genetic findings: case series. Swiss Med Wkly 2006;136: 189-93.

14. Beggs AH, Hoffman EP, Snyder JR, Arahata K, Specht L, Shapiro F, \& al. Exploring the molecular basis for variability among patients with Becker Muscular Dystrophy: Dystrophin gene and protein studies. Am J Hum Genet 1991;49:54-67.

15. Winnard AV, Klein CJ, Coovert DD, Prior T, Papp A, Snyder P, \& al. 
Characterization of translation frame exception patients in Duchenne/ Becker muscular dystrophy. Hum Mol Genetics 1993;2:737-44.

16. Fanin M, Freda MP, Vitiello L, Danieli GA, Pegoraro E, Angelini C. Duchenne phenotype with in-frame deletion removing major portion of dystrophin rod: Threshold effect for deletion size? Muscle Nerve 1996;19:1154-60.

17. Nevo Y, Muntoni F, Sewry C, Legum C, Kutai M, Harel S, \& al. Large in frame deletions of the rod-shaped domain of the dystrophin gene resulting in severe phenotype. Isr Med Assoc J 2003;5:94-7.

18. Singh V, Sinha S, Mishra S, Chaturvedi LS, Pradhan S, Mittal RD, e al. Proportion and pattern of dystrophin gene deletion in North Indian Duchenne and Becker Muscular dystrophy patients. Human Genetics 1997;99:206-8.

19. Banerjee M, Verma IC. Are there ethnic differences in deletions in the dystrophin gene? Am J Med Genet 1997;68:152-7.

20. Mital A, Kumari D, Gupta M, Goyle S. Molecular characterisation of
Duchenne muscular dystrophy and phenotypic correlation. J Neurol Sci 1998;157:179-86.

21. Kumari D, Mital A, Gupta M, Goyle S. Deletion analysis of the dystrophin gene in Duchenne and Becker muscular dystrophy patients: Use in carrier diagnosis. Neurol India 2003;51:223-6.

22. Dastur R, Gaitonde P, Khadilkar S, Nadkarni J. Deletion analysis of Duchenne/Becker muscular dystrophy gene using multiplex polymerase chain reaction. Ann Indian Acad Neurol 2004;7:333-7.

23. Basak J, Dasgupta UB, Banerjee TK, Senapati AK, Das SK, Mukherjee SC. Analysis of dystrophin gene deletions by multiplex PCR in eastern India. Neurol India 2006;54:310-11.

Accepted on 12-03-2008

Source of Support: Nil, Conflict of Interest: None declared. 